\title{
Combination set to transform HCC therapy
}

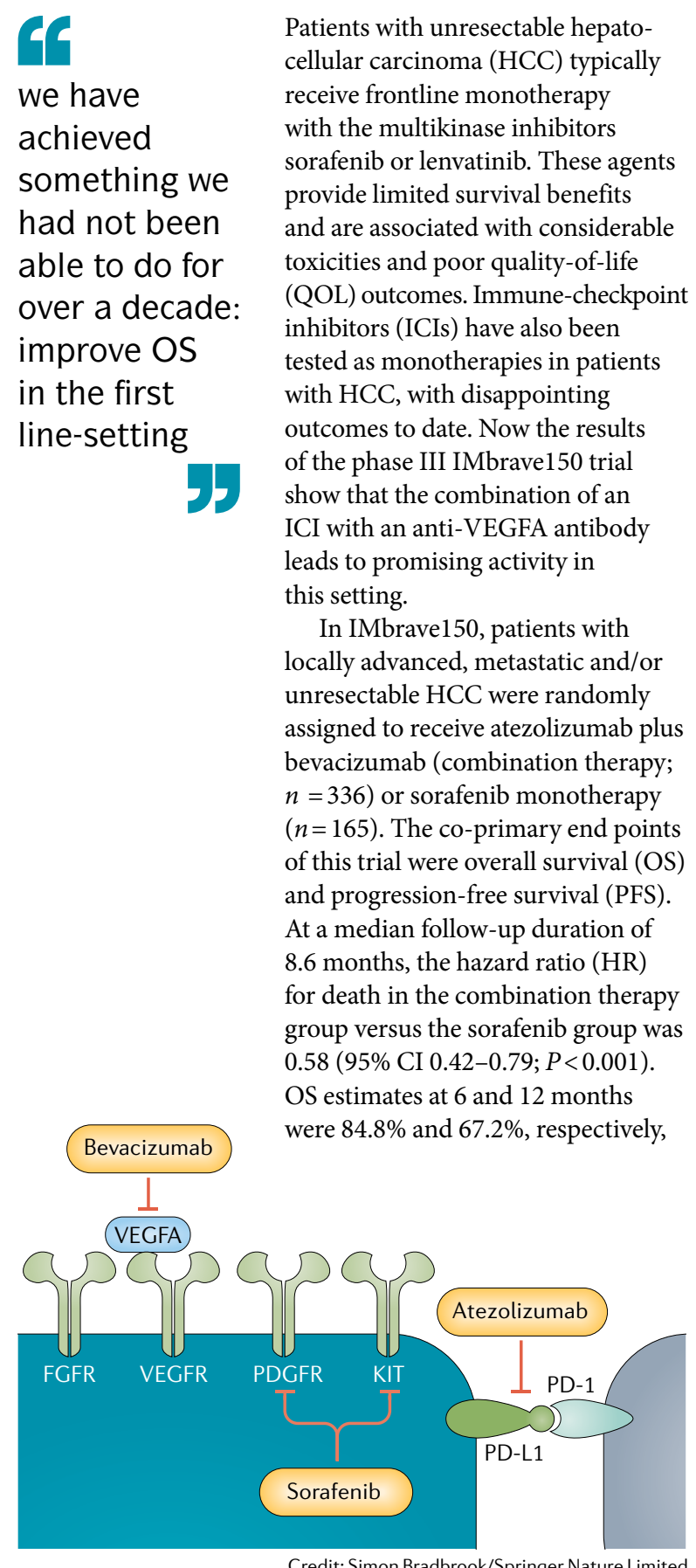

with combination therapy, and $72.2 \%$ and $54.6 \%$ with sorafenib. Median PFS durations were longer in patients receiving combination therapy than with sorafenib: 6.8 months versus 4.3 months (HR 0.59, 95\% CI $0.47-0.76 ; P<0.001)$. At 6 months, PFS was $54.5 \%$ and $37.2 \%$, respectively. The OS and PFS benefits were consistent across clinically defined subgroups. The confirmed objective response rates were $27.3 \%$, including complete responses (CRs) in 5.5\%, with combination therapy and $11.9 \%$, with no CRs, with sorafenib.

The median time to deterioration of patient-reported QOL, evaluated using the EORTC QLQ-C30 questionnaire, was longer for patients receiving combination therapy (11.2 months versus 3.6 months; HR 0.63 , 95\% CI 0.46-0.85). The incidence of adverse events (AEs) of any grade was similar in both groups: $98.2 \%$ and $98.7 \%$. Serious AEs were more frequent with combination therapy (38.0\% versus $30.8 \%$ ), although the difference was not $\geq 2 \%$ for any AE. The most common grade 3-4 AE in patients receiving combination therapy was hypertension: $15.2 \%$ versus $12.2 \%$ with sorafenib. AEs led to treatment discontinuation in $15.5 \%$ of patients receiving combination therapy (with 7\% discontinuing both treatments) and $10.3 \%$ of those receiving sorafenib.

"The results of IMbrave150 constitute an important milestone in our progress against this deadly disease. In addition, the favourable $\mathrm{AE}$ profile with this combination is really the icing on the cake," explains co-lead investigator Richard Finn. "This study represents a breakthrough comparable to the survival benefit of sorafenib over placebo presented in 2008," opines Josep Llovet, who was not involved in this study. He adds: "these results pose this combination as the first and only treatment superior to sorafenib so far, and thus, as the recommended standard first-line therapy for patients with HCC."

The results of IMbrave150 differ from those of previous clinical trials of ICIs in HCC. "The negative results of a trial comparing nivolumab with sorafenib in the first line of treatment and another comparing pembrolizumab with placebo in the second line represented an important drawback for the HCC community," states Llovet. "We know that singleagent ICIs are beneficial for a subset of patients, but the end points of randomized studies were not met," explains Finn, adding: "In this study, we have doubled the response rate of single-agent ICI. By doing so, we have achieved something we had not been able to do for over a decade: improve OS in the first line-setting."

The therapeutic activity of ICIs might be enhanced through combinations with other agents that promote antitumour immunity. In IMbrave150, this effect has been achieved with the incorporation of the anti-VEGFA antibody bevacizumab. Various combinations are currently being tested in phase III trials in the frontline setting. "Whether the combinations tested provide even better results will depend upon the capacity of specific kinase inhibitors and/or ICIs to transform immunologically 'cold' tumours into 'hot' tumours," Llovet concludes.

Diana Romero

ORIGINAL ARTICLE Finn, R. S. et al. Atezolizumab plus bevacizumab in unresectable hepatocellular carcinoma. N. Engl.J. Med. 382, 1894-1905 (2020) 